\title{
Editorial: Novel Diagnostic and Therapeutic Strategies for Liver Cancer
}

Liver cancer is the third leading cancer worldwide, with an increasing mortality rate accounting for 700,000 deaths every year. ${ }^{1}$ It is the fifth most common cancer in men and the ninth most common cancer in women. ${ }^{1}$ As estimated by the American Cancer Society, in the year 2020, about 42,810 new cases of liver cancer were diagnosed and about 30,160 people died as a result. Despite advanced technologies in the research field, the prognosis rate is very low as is the five-year survival rate. ${ }^{1}$ This mortality burden is aggravated by the western lifestyle, which is associated with varied risk factors for liver cancer. These risk factors include alcohol, smoking, and obesity. Other contributing factors are chronic viral hepatitis, cirrhosis, nonalcoholic fatty liver disease (NAFLD), and type 2 diabetes. $^{2}$

Conventional therapies, including surgery and chemoradiotherapies, are beneficial to the patient, but they are limited due to late diagnosis and tumor recurrence. Moreover, overexpression of various growth factors and dysregulated transcription factors, including STAT, AP-1, HIF-1 $\alpha$, and NF- $\kappa$ B, plays a major role in disease recurrence. In this volume, contributors explore novel diagnostic and therapeutic strategies and novel biomarkers that have been found beneficial for patients.

Despite advanced technologies in the cancer research field, incidence and mortality rates for liver cancer remain very high. Therefore, early diagnosis to improve survival and disease management requires immediate attention. Cancer biology researchers are now identifying unique molecular candidates like proteomics and genomic profiles for a better understanding of the biological signatures associated with liver cancer. Early-state differential diagnosis through these signatures can remarkably improve patient survival. Ghanta et al. and Tekupalli et al. focus on recent developments in the targeting of cardiac biomarkers, serum biomarkers, growth factors, and the like, for early-stage HCC diagnosis leading to increased survival rates.
Additionally, researchers are focusing on the importance of lncRNAs because they play a mediator role in liver cancer pathogenesis and metastasis. Recent studies have revealed that dysregulated lncRNAs are involved in tumor pathogenesis and promote cell proliferation, migration, invasion, EMT, and drug resistance. ${ }^{3-5}$ Thus, they have potential as targets and biomarkers for tumor diagnosis and prognosis. Malla discusses recent updates on lncRNA targeting in liver cancer diagnosis and therapeutic strategies, presenting overall novel perspectives. Clinical practices encompass evaluation of biomarkers like growth factors, circulating nucleic acids, lncRNAs, and cardiac markers, which aid diagnosis of liver cancer in its early stages.

One way in which liver cancers arise is through disorders like NAFLD, a leading cause of chronic liver diseases such as nonalcoholic steatohepatitis, which can progress into hepatocellular carcinoma. ${ }^{6}$ The ultimate cause of liver cancer is increased fat deposits in the liver. In order to combat this pathological condition, it is essential that NAFLD be understood in order to understand routine lipid metabolism in the liver under varied altered conditions. In this respect, Mungamuri et al. address altered underlying mechanisms involved in hepatic lipid uptake and fatty acid oxidation. They also discuss modified signaling pathways that cause accumulation of excess fat in the liver, along with dysregulated transcription factors and other lipolytic enzymes. Such a comprehensive understanding of changes in the metabolism of lipids may lead to successful therapies against NAFLD. The transcription factors discussed play a key role in regulating hepatic de novo lipolysis, and in altering lipid progression during NAFLD. They include NF- $\kappa$ B, STAT, HIF- $1 \alpha$, and AP-1 mediated signaling, which are regulators of cell survival, immunity, and inflammation. Dysregulated, these cascades promote invasion and metastasis in liver carcinogenesis. 
Aliya explores the activation of transcription factors involved in progression and invasion, along with the FDA-approved drugs lenvatinib, regorafenib, and tyrosine kinase inhibitors that target transcription factors to control tumor progression. Altered expression of some transcription factors along with growth factors causes an imbalance of cellular energy and can thereby drive tumorigenesis. Proliferation, invasion, and metastasis occur through multiple signaling pathways such as activation of the PI3K/Akt/mTOR axis, which is stimulated by the binding of growth factors to their receptors. These pathways further induce cell proliferation, survival, angiogenesis, and metastasis. The growth factors EGF, VEGF, and IGF underlie activation of angiogenesis, which eventually promotes metastasis in liver cancer. ${ }^{7}$ During injury or damage, mature hepatocytes upregulate varied growth factors for liver regeneration. However, transient upregulated growth factors are dysregulated in these hepatocytes and thus play a crucial role in hepato-carcinogenesis. Muppala et al. explores proangiogenic growth factors and their underlying mechanisms that promote angiogenesis in liver cancer. Their review highlights the regulation of angiogenesis and metastasis via the targeting of growth factor signaling pathways for effective HCC therapy.

Altogether, this volume provides an in-depth understanding of the novel therapeutic and diagnostic options currently available. It also explores current advancements in and applications for the diagnosis and therapy of liver cancer. It is my immense pleasure to present this comprehensive summary of novel therapeutic strategies to the scientific community for the benefit of patients suffering from liver cancer.

\section{REFERENCES}

1. Siegel RL, Miller KD, Goding Sauer A, Fedewa SA, Butterly LF, Anderson JC, Cercek A, Smith RA, Jemal A. Colorectal cancer statistics, 2020. CA Cancer J Clin. 2020;70(3):145-64.

2. Smith RA, Andrews KS, Brooks D, Fedewa SA, Manassaram-Baptiste D, Saslow D, Brawley OW, Wender RC. Cancer screening in the United States, 2018: A review of current American Cancer Society guidelines and current issues in cancer screening. CA Cancer J Clin. 2018;68(4):297-316.

3. Xu Y, Zhang X, Hu X, Zhou W, Zhang P, Zhang J, Yang S, Liu Y. The effects of lncRNA MALAT1 on proliferation, invasion and migration in colorectal cancer through regulating SOX9. Mol Med. 2018;24(1):1-15.

4. Liu M, Zhang H, Li Y, Wang R, Li Y, Zhang H, Ren D, Liu $\mathrm{H}$, Kang C, Chen J. HOTAIR, a long noncoding RNA, is a marker of abnormal cell cycle regulation in lung cancer. Cancer Sci. 2018;109(9):2717-33.

5. Feng C, Xian Q, Liu S. Micro RNA-518 inhibits gastric cancer cell growth by inducing apoptosis via targeting MDM2. Biomed Pharmacother. 2018;97:1595-602.

6. Younossi Z, Stepanova M, Ong JP, Jacobson IM, Bugianesi E, Duseja A, Eguchi Y, Wong VW, Negro F, Yilmaz Y. Nonalcoholic steatohepatitis is the fastest growing cause of hepatocellular carcinoma in liver transplant candidates. Clin Gastroenterol Hepatol. 2019;17(4):748-55. e3.

7. Wei G, An P, Vaid KA, Nasser I, Huang P, Tan L, Zhao S, Schuppan D, Popov YV. Comparison of murine steatohepatitis models identifies a dietary intervention with robust fibrosis, ductular reaction, and rapid progression to cirrhosis and cancer. Am J Physiol Gastrointest Liver Physiol. 2020;318(1):G174-G88.

\section{Guest Editor:}

Ganji Purnachandra Nagaraju, PhD, DSc, FAACC Department of Hematology and Medical Oncology Winship Cancer Institute, Emory University

Atlanta, Georgia 